\title{
Elementos de integración de microempresas comerciales en el oriente del Estado de México en los primeros años del siglo XXI
}

\author{
Nidia López Lira*
}

\section{Resumen}

En México la cultura de la integración empresarial es casi nula sobre todo en los negocios de menor tamaño. Este artículo analiza algunos elementos que favorecerían o dificultarían un proyecto de integración de los microempresarios dedicados al comercio en un municipio de la zona oriente del Estado de México, en el marco de un modelo de asociación empresarial del gobierno federal. El municipio de Valle de Chalco Solidaridad se constituyó en la última década del siglo XX; se caracterizó desde sus inicios por la disponibilidad de sus habitantes para unirse y lograr beneficios comunes, con él inició el Programa Nacional de Solidaridad de Carlos Salinas de Gortari en 1989. Este hecho podría ser un elemento a favor para el desarrollo de proyectos de integración empresarial; sin embargo, existen otros que podrían considerarse en contra, tales como el bajo nivel educativo y económico de sus habitantes y la atomización de los giros económicos, características compartidas de las comunidades suburbanas del oriente del Estado de México en los primeros años del siglo XXI: a poco más de una década de la entrada en vigor del TLCAN y después de al menos tres sexenios presidenciales caracterizados por la aplicación abierta de un modelo económico neoliberal.

Palabras clave: integración, programas de apoyo gubernamentales.

\section{Antecedentes}

Valle de Chalco Solidaridad era en sus orígenes un enorme asentamiento humano irregular, iniciado aproximadamente en 1978, cuyos habitantes carecían de servicios públicos básicos, su nivel económico era bajo y la mayoría de ellos provenía

* Profesora de la Unidad Académica profesional Valle de Chalco de la Universidad Autónoma del Estado de México. Correo electrónico: nidia_lopez_lira@yahoo.com.mx 
del interior de la República o había sido expulsada de la Ciudad de México. Su cercanía con el D.F. lo convirtió — hasta la fecha - en un espacio propicio para personas en busca de cualquier oportunidad de subsistencia y de mejora en el nivel de vida. En 1989 el presidente Carlos Salinas de Gortari puso en marcha el Programa Nacional de Solidaridad (PRONASOL) con los pobladores de aquella época, con ello se convirtió en ese sexenio en modelo de trabajo y desarrollo comunitario. Actualmente, a la mitad de la primera década del siglo XXI, el municipio es muestra de una comunidad emergente con una problemática representativa de la aplicación indiscriminada del modelo neoliberal: marginación, falta de empleo que se genera en el propio municipio, pobreza, insalubridad, delincuencia, entre otros.

El municipio se empezó a gestar en la penúltima década del siglo XX y alcanzó su formalización en la última de ese siglo; en la primera del siglo XXI ha logrado avances, pero también estancamientos tanto en el ámbito social como en el económico, lo cual es importante analizar.

Cabe señalar que la Secretaría de Economía ha diseñado programas que están enfocados a hacer más competitivas a las empresas de menor tamaño, entre ellos ha incluido algunos que se basan en la integración empresarial y que contemplan a las universidades como organismos intermedios entre el gobierno y las empresas.

No es privativo de este municipio la escasa utilización de programas de apoyo dirigidos al fortalecimiento de las empresas, misma que podría ser ocasionada por falta de información y de cultura empresarial, desconfianza, etc., aunque se desconocen las causas específicas. A raíz de lo anterior, surgió la pregunta ¿qué elementos a favor y qué elementos en contra existen en los primeros años del siglo XXI en Valle de Chalco Solidaridad para desarrollar proyectos de integración de empresas comerciales y lograr una mayor fortaleza económica?

En este artículo se abordan los elementos a favor y en contra al iniciar un proyecto de integración entre las empresas comerciales de menor tamaño del municipio, además, se agrega la variable de contar con una institución de educación superior como líder del proyecto. Para desarrollar lo anterior, este trabajo se dividió en los siguientes apartados: en el primer apartado, metodología, se describe el tipo de investigación realizada para obtener información, la determinación de la población y el cálculo de la muestra; en el siguiente apartado se hace una revisión teórica de los temas integración, microempresa y cultura de solidaridad en el 
municipio; el tercero describe las características de las empresas y de la población municipal; en el cuarto se presentan las características de las instituciones de educación superior de la región; el quinto se destina a hacer una revisión de los programas de apoyo gubernamentales, centrando la atención en aquellos que contemplan estrategias de integración; finalmente, en el último apartado se efectúa un análisis de los elementos a favor y en contra de un proyecto de integración empresarial en el municipio, retomando la información analizada en los apartados anteriores con una visión en conjunto de población, empresas, gobierno e instituciones educativas; asimismo, se presentan algunas conclusiones y propuestas que podrían apoyar al diseño del proyecto.

\section{Metodología}

La información que sirvió de base para elaborar este artículo se obtuvo de dos investigaciones relativas al tema: la parte medular la aportó un proyecto de investigación desarrollado por la autora con el apoyo de los integrantes del Cuerpo Académico al que pertenece; los datos relativos a las instituciones de educación superior (IES) de la región los aportó, principalmente, la tesis de maestría de la autora. La metodología utilizada en el proyecto fue de dos tipos:

- Investigación documental. A través de ella se hizo una revisión e interpretación de los datos estadísticos del Instituto Nacional de Geografía e Informática (INEGI) de los años 2000 y 1995; con esto se obtuvo una descripción del entorno en cuanto a población y actividad empresarial en la región.

- Investigación de campo. Se diseñó empíricamente un cuestionario para conocer cuáles serían los principales motivos por los cuales los microempresarios del municipio participarían en el proyecto, o no (ver Anexo 1); se obtuvo una muestra de 372 empresas de una población de 3,784 unidades de negocio; el criterio de exclusión para la determinación de la población fue: "Empresas comerciales incluidas en el padrón del Municipio de Valle de Chalco Solidaridad que no sean entidades de apoyo social (asistencia pública o privada)". La muestra se eligió a través de muestreo probabilístico con una probabilidad de ocurrencia de $90 \%$ y un error estándar de 0.015 mediante el método de selección sistemática de elementos muestrales ${ }^{1}$. Se clasificó a las empresas por giro para obtener una muestra probabilística estratificada, con el interés de garantizar que todos los giros,

\footnotetext{
1 Roberto Hernández Sampieri, Metodología de la investigación, pp. 309-311.
} 
independientemente del número de unidades de negocio con el que contaran tuvieran la misma probabilidad de ser seleccionados. La muestra probabilística $(n)$ de las empresas estratificada por giros y el número de cada estrato se determinó a través del cálculo de un coeficiente de estratificación ${ }^{2}$; en el caso de las instituciones de educación superior de la región, no fue necesario aplicar ninguna técnica de muestreo ya que sólo existe una en el municipio, pero - con el objeto de tener al menos dos opiniones diferentes - se decidió indagar en el municipio aledaño de Chalco y contar con datos de más de una institución. Asimismo, se aplicó una entrevista guiada a los directores de dos IES para obtener información relativa a la misión, visión, así como la disponibilidad de las mismas para participar en un proyecto de integración empresarial en la zona (ver anexo 2).

\section{Consideraciones teóricas}

\section{Integración}

Se entiende por integración a la acción de unirse — no necesariamente en términos contractuales, sino operativamente - dos o más entidades con el fin de hacer frente a distintas situaciones que ponen en riesgo su estabilidad en el mercado; algunos autores llaman a este fenómeno cooperación. Las estrategias de desarrollo, que tienen como finalidad el fortalecimiento o el crecimiento de una empresa o área de la misma, son múltiples y pueden poseer características muy diferentes entre sí porque se establecen para resolver situaciones específicas de cada empresa en particular. Diversas estrategias de desarrollo podrían enumerarse; sin embargo, el universo es amplio porque consideran necesidades y características particulares de las empresas, de los productos/servicios, de los mercados, etc. Lo que sí se puede afirmar es que las estrategias de asociación tienen como finalidad el mejorar el desarrollo de los participantes de tal forma que la literatura referente a modelos de asociación aborda casi de manera implícita las estrategias de desarrollo.

Existen diferentes elementos que determinan el tipo de integración que se da entre las empresas; por ello, es posible identificar cierta dirección y profundidad de la misma. El especialista Hubert Schmitz sostiene que las situaciones riesgosas a enfrentar por las empresas son puntos de inflexión que determinarán el nivel y tipo de cooperación que adoptarán; también menciona una clasificación de acuerdo con lo que ha observado en cuatro clusters industriales de América Latina y Asia:

Idem. 
Es posible identificar diferentes formas y niveles de integración. La forma más básica de representar la cooperación es de manera lineal hacia atrás o hacia delante: Cuando los fabricantes deciden establecer nexos más estrechos con los proveedores locales de insumos, se dice que es una cooperación hacia atrás, o bien pueden estrechar nexos con los distribuidores de su producto, en cuyo caso recibe el nombre de integración hacia delante. ${ }^{3}$

\section{Tabla 1}

Formas de cooperación interempresarial

\begin{tabular}{|c|c|c|}
\hline Cooperacida & Bilateral & Mufillateraf \\
\hline Horizontal & Se comfarte el equipo & Asociacićn sectorial \\
\hline Vertical & $\begin{array}{l}\text { Mejoras en los ecengenentes por parte } \\
\text { del productor y del utilizador }\end{array}$ & $\begin{array}{l}\text { Aliarza en tomo a una cadena de } \\
\text { valor loed }\end{array}$ \\
\hline
\end{tabular}

Fuente: "¿Tiene importancia la cooperación Local? Experiencias de clusfers industriales en el sur de Asia y América Latina*, en El Mercado de Valores, No، 9/septiembre, México, Nacional Financiera, 2000.

De igual forma, resulta posible identificar distintos modelos de asociación empresarial que van enfocados a lograr distintas estrategias de desarrollo, por ejemplo:

\section{- Cadenas productivas}

Las cadenas productivas son un conjunto de procesos interrelacionados que intervienen en la incorporación de valor a las materias primas para la obtención de bienes y servicios que llegan al consumidor final. Una definición más amplia indica que las cadenas productivas "[...] son todas las operaciones que se inician con la disposición de materia prima hasta la entrega del producto al cliente final, incluidos los flujos de información". ${ }^{4}$ Las cadenas productivas llevan implícito el factor cooperación interempresarial; contemplan el hecho de que las empresas pueden o no estar cerca geográficamente, que incluyen una estructura de poder y que se desenvuelven en un ambiente institucional, que podrían ser por ejemplo un Gobierno, una Asociación o una Universidad.

\section{- Empresas integradoras}

\footnotetext{
3 Hubert, Schmitz, "¿Tiene importancia la cooperación Local? Experiencias de clusters industriales en el sur de Asia y América Latina", en El Mercado de Valores, p. 7.

4 Flávia Gutiérrez Motta, www.prd.usp.br
} 
La empresa integradora debe prestar servicios especializados de diversa índole a las integradas, los cuales pueden abarcar o no actividades del proceso productivo; la integradora es jurídicamente independiente a las integradas, pero su capital es propiedad de estas últimas. Al respecto Isabel Rueda señala:

El principal objeto de constituir una empresa integradora es el de elevar la competitividad de las asociadas (que preferentemente son de escala micro, pequeña y mediana), consolidar su presencia en el mercado nacional e incrementar su participación en las exportaciones. ${ }^{5}$

\section{- $\quad$ Clusters o distritos industriales}

Se entiende por cluster industrial a la aglomeración espacial de empresas dedicadas a la misma o similar actividad y donde se dan múltiples relaciones entre las entidades que lo conforman. En relación con esto, Alfred Marshall describió "[...] los distritos industriales hace más de un siglo como una aglomeración territorial de empresas del mismo ramo o de ramos similares, en donde la mano de obra especializada, los insumos y la prestación de servicios están fácilmente disponibles, y en donde las innovaciones se difunden rápidamente." ${ }^{6}$

\section{- $\quad$ Redes de empresas}

Es una integración en la que se establecen relaciones de largo plazo entre las empresas para realizar actividades expansionistas que en lo individual sería difícil lograr. También se le define de la siguiente manera:

Una forma organizacional de red, según Podolny y Page (1998), es una colección de actores (más de uno) que establecen relaciones de cambio de largo plazo, y que a un mismo tiempo no poseen legitimidad y autoridad para arbitrar y resolver disputas que pueden ocurrir durante el cambio. Las empresas de una red cooperan en el desarrollo de proyectos comunes, una complementando a otra en especialización, para resolver problemas, conquistar nuevos mercados, alcanzar escala, y otros objetivos que van de acuerdo a los alcances individuales de las empresas (Ceglio y Dini, 1999) Algunas formas de redes son: joint ventures, alianzas estratégicas, grupos de negocios, franquicias, consorcios de investigación, contratos relacionados, acuerdos de outsourcing, etc. ${ }^{7}$

5 Isabel Rueda Peiro et al., Las empresas integradoras en México, p. 10.

6 Jörg Meyer-Stamer, "Estrategias de desarrollo local y regional: clusters, política de localización y competitividad sistémica", en El mercado de valores, p. 19. 


\section{Caracterización de las microempresas, su importancia y casos de integración}

Mucho se ha hablado de las características de las microempresas en México, desde el número de empleados con los que cuentan, hasta los problemas que más comúnmente enfrentan y que son tan constantes que más que problemas para su desarrollo, circunscritos en un marco espacio-tiempo determinado, se han vuelto parte de sus características. Una de las principales problemáticas de las microempresas en México ha sido su gran limitación para acceder a oportunidades de crédito en el sistema financiero tradicional. Este problema lo determina una serie de características que no les permite ser consideradas sujetos de crédito por el riesgo que éstas traen implícito. Algunas de ellas son:

1. Se constituyen con poca inversión.

2. Utilizan sencillos procesos productivos, de distribución y de comercialización.

3. En muchas actividades se desconoce la existencia de procedimientos o técnicas innovadoras como consecuencia de la falta de recursos para inscribirse en los centros de divulgación industrial.

4. Es una constante la falta de recursos para capacitación tanto de operarios como de ejecutivos y de directivos relacionados con los nichos de mercado descubiertos o con nuevos productos y servicios.

5. Entre estos empresarios no existe un monitoreo permanente de los mercados, por lo que programan su producción y comercialización a corto plazo.

6. No tienen una visión de largo plazo sobre las inversiones a realizar para acceder competitivamente a los mercados, como tampoco del mantenimiento y reposición correspondiente de su "aparato productivo, distributivo y comercializador".

Tales características provocan un círculo vicioso porque les impide acceder fácilmente al crédito porque son riesgosas y su rentabilidad es limitada, situaciones que ocurren en gran medida porque carecen del impulso que genera el crédito.

Flávia Gutiérrez Motta, www.prd.usp.br

8 Genaro Sánchez Barajas, Ponencia "El desarrollo regional de las micro y pequeñas empresas mexicanas", www.monografías.com/trabajos28/desarrollo_pymes_mexicanas/desarrollo_pymes_mexicanas.shtml, 2006. 
Tabla 2

Definición de MIPyMES atendiendo al número de empleados

\begin{tabular}{|c|c|c|c|}
\hline TAMANOO & IVDUSTRIA & COMERCTO & SERVICTE \\
\hline Micno enipresa & $0-10$ & $0-10$ & $0-10$ \\
\hline Pequeta enteresa & $11-50$ & $11-30$ & 11.50 \\
\hline Mediana empiesa & 51.259 & 31.100 & 51.100 \\
\hline
\end{tabular}

\section{Fuente: Fracción III articulo 3 de la Ley para el Desarrollo de la Competitividad de las micro, pequeña y mediana empresas, 2005}

Otra de sus características esenciales es la baja competitividad y la falta de generación de empleos bien remunerados, por lo que se constituyen como negocios de subsistencia para sus propietarios. No obstante lo anterior, las microempresas son importantes porque alivian hasta cierta medida la pobreza extrema y representan, por lo tanto, la oportunidad de mejorar el nivel de vida de sus propietarios. Adicionalmente, diversas fuentes establecen que en México entre el 80 y el 90\% de las empresas son micro, pequeña y mediana, lo que las convierte en elemento clave de activación de la economía interna del país. Economías más sólidas en el nivel mundial han logrado fortalecer a las empresas de menor tamaño a través de eslabonamientos productivos entre ellas mismas y con las empresas grandes, lo que ha elevado considerablemente su competitividad. Revisando las conclusiones de las investigaciones realizadas por Jorge Carrillo y Alfredo Hualde (2000) en el cluster de la electrónica en Tijuana y por Hubert Schmitz (2000) en un cluster de calzado en Guadalajara, es posible identificar algunos elementos que han favorecido u obstaculizado la colaboración empresarial:

Tabla 3

Ejemplos de elementos a favor y en contra de asociación en dos clusters mexicanos

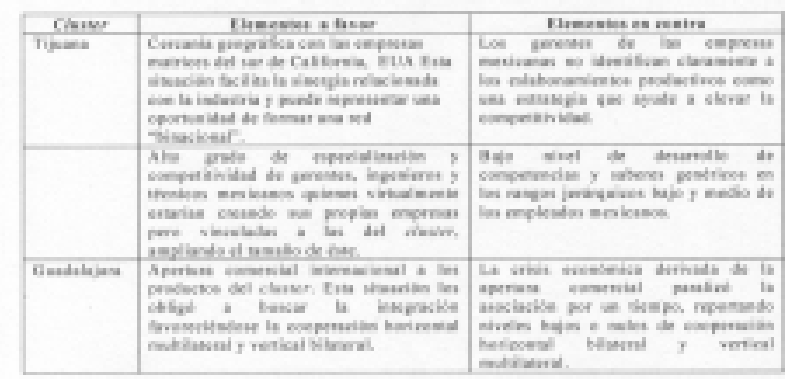

Fuente: Elaboración propia con datos de las investigaciones citadas 
Éstos son sólo algunos ejemplos de cómo una variedad de circunstancias pueden constituirse en elementos favorables o desfavorables para la integración. En el caso de Valle de Chalco, un elemento que vale la pena resaltar es su tradición de solidaridad.

\section{Cultura de solidaridad en Valle de Chalco}

Sería muy aventurado decir que existe una cultura empresarial de solidaridad en el municipio; sin embargo, sí es posible hablar de una unión entre los habitantes para hacer frente a problemas específicos.

De esta tradición de solidaridad dan cuenta las historias de pobladores originales de Xico (nombre de la región donde ahora se encuentra Valle de Chalco Solidaridad y que ya era habitada desde la época prehispánica), cuando al ser trasladados coercitivamente de sus tierras por Iñigo Noriega, empresario de la época del porfiriato:

la estrategia que asumieron tanto el pueblo de Xico, como los demás pueblos de las márgenes del lago, fue emprender en primera instancia una batalla legal en los tribunales contra las afectaciones ocasionadas por el empresario a causa de la desecación lacustre y en segunda instancia participaron activamente en la rebelión campesina de Emiliano Zapata, a diferencia de otros pueblos de la región. ${ }^{9}$

Así como las historias de los propios inmigrantes:

este enorme asentamiento que poco a poco se va convirtiendo en una ciudad con todos sus servicios e instituciones, al principio contó con el infatigable trabajo de las mujeres del valle... lavando ropa, persiguiendo a las pipas, haciendo la comida... integrando los comités de construcción de las primeras escuelas [...] Del miedo inicial que teníamos a los vecinos, la buena vecindad no ha hecho sino crecer, ha habido mucha solidaridad ante la desgracia ajena, al enfrentar la violencia circundante, de la que siempre nos tocó ver sus efectos desastrosos. ${ }^{10}$

Se sabe que los vecinos estaban organizados en diferentes comités de obras: de escuelas, de guarniciones y banquetas y de lecherías, trabajando así para lograr mejorar el precario nivel de vida en el que se encontraban.

9 Alejandro Tortolero, "Haciendas, pueblos y gobierno porfirista: los conflictos por el agua en la región de Chalco", citado por Jaime Loyola Rocha, en Historias para ser contadas a los recién llegados, p. 49.

${ }^{10}$ Jaime Noyola Rocha, Historias para ser contadas a los recién llegados, Municipio de Valle de Chalco Solidaridad, México, 2003, pp. 54-55. 


\section{Caracterización de las microempresas y de la población de Valle de Chalco Solidaridad}

En 1999 el perfil empresarial del municipio era definitivamente comercial, tomando como criterio para esta afirmación el mayor número de unidades de negocio. Esta información nos permitió decidir que la propuesta de asociación empresarial se inclinaría a integrar a los empresarios del comercio para liberar presión por el exceso de competencia y se encaminaría a buscar nichos de mercado.

Tabla 4

Unidades económicas por sector (1999)

\begin{tabular}{|c|c|c|c|c|c|c|}
\hline Manicipie & $\begin{array}{l}\text { Validades } \\
\text { del sector } \\
\text { comereis }\end{array}$ & $\%$ & $\begin{array}{l}\text { Unidades } \\
\text { del secter } \\
\text { servicios }\end{array}$ & * & $\begin{array}{l}\text { Unislades } \\
\text { del sector } \\
\text { indectria }\end{array}$ & $\%$ \\
\hline Valle de Chales & $5, \$ 80$ & $65 \%$ & 2374 & $25 \%$ & 887 & $10 \%$ \\
\hline
\end{tabular}

Nota: El porcentaje se determinó con relación al total de unidades económicas del municipio

Fuente: Elaboración propia con datos del INEGI

Tabla 5

Valor agregado censal bruto por sector en miles de pesos, 1999

\begin{tabular}{|c|r|r|r|r|}
\hline Municipio & \multicolumn{1}{|c|}{ Comercio } & \multicolumn{1}{|c|}{ Servicios } & \multicolumn{1}{c|}{ Industria } & \multicolumn{1}{c|}{ Total } \\
\hline Valle de Chalco & 213,407 & 62,636 & $-11,710$ & 287,753 \\
\hline
\end{tabular}

Fuente: Elaboración propia con datos del INEGI

El municipio mostraba, por lo tanto, un fenómeno similar a lo que ocurre no sólo en el pais, sino en la mayoria de los paises en vías de desarrollo de la actualidad: una acentuación de su economia al sector comercial en detrimento del sector servicios y sobre todo del sector industrial.

Tabla 6

Personal ocupado, remuneraciones, ingresos y activos

fijos por sector (1999)

\begin{tabular}{|c|c|c|c|c|}
\hline Veriable par Maniopis & Secter Ceasercis & $\begin{array}{l}\text { Secior } \\
\text { Iatestria }\end{array}$ & Sester Sorrikias & Totall \\
\hline Fersmel ecaysobo & & $3,00 x$ & 4000 & [s:21] \\
\hline 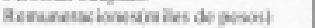 & 65.135 & 13.3.35 & 12.791 & Ist.joi \\
\hline 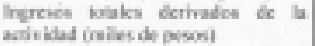 & $1,675,127$ & 51,219 & 134.937 & $2,201,083$ \\
\hline Actives fios inile de peres) & 212.255 & 254.796 & 105.041 & $\operatorname{sen} 60$ \\
\hline
\end{tabular}

Fuente: Elaboración propia con datos del INEGI 
Por su parte, el instrumento de recolección de datos aportó la siguiente información de las empresas comerciales del municipio:

\section{- Empleados}

El número promedio de empleados con los que contaban las empresas comerciales de Valle de Chalco era entre uno y dos; se encontraban en esta situación el 97\% de la muestra y el 3\% restante contaba con cinco empleados como máximo. Este resultado es un indicador de que las empresas son micro ${ }^{11}$ y que la gran mayoría no emplea personal, sino que son los mismos dueños quienes atienden sus negocios.

\section{- $\quad$ Pertenencia a asociaciones de comerciantes}

El 70\% de las empresas encuestadas no se encontraban afiliadas a alguna asociación de comerciantes. El 30\% de unidades de negocio que manifestó estar afiliado a alguna asociación recibía servicios de gestión de trámites y asesoría, pero las agrupaciones que mencionaron estos comerciantes pertenecían de manera directa o indirecta a alguna agrupación política municipal.

\section{- Utilización de programas de apoyo}

Era casi nula la utilización de programas de apoyo a la microempresa, de ello hablan el 97\% de los negocios que afirmaron no participar en ningún tipo de programa, ya fuera municipal, estatal o federal. No tenían claro qué necesitarían para participar en un programa de apoyo para el crecimiento de su empresa, así lo expresó abiertamente el $29 \%$ de los encuestados, contra un $24 \%$ que opinó que necesitaba mayor información para decidir si participar o no, $11 \%$ afirmó no necesitar nada, $6 \%$ indicó necesitar financiamientos y el resto indicó necesitar tiempo, mayor confianza, o bien que no le interesaba ese tipo de apoyos.

\section{- Disponibilidad para participar en un programa de apoyo gubernamental}

El 62\% de los encuestados manifestaron estar interesados en participar en un programa de apoyo gubernamental a las microempresas, sin embargo, con las respuestas del indicador anterior se observa que no saben con precisión lo que pretenderían al participar en el supuesto programa.

\footnotetext{
${ }^{11}$ Según el criterio de clasificación de la Secretaría de Economía.
} 
- $\quad$ Volumen mensual de sus compras y sus gastos

El rango de compras del $73 \%$ de los encuestados se encontraba entre $\$ 1,000$ y menos de \$3,000 pesos con gastos de transportación de hasta \$300.

- $\quad$ Registro contable de sus operaciones

El 38\% manifestó no llevar ningún tipo de registro contable. El 37\% manifestó llevar registros contables en un cuaderno. El 25\% restante utiliza los servicios de un contador público.

- Interés de colaborar con otros comerciantes

El 56\% de los encuestados manifestaron estar dispuestos a colaborar con otros comerciantes porque esperarían ayuda y desarrollo para sus negocios, fortalecimiento y unión y homologar intereses económicos. El 44\% restante no estaría dispuesto a colaborar con otros debido a que pensaban que les quitaría mucho tiempo un proyecto de este tipo y que había demasiada competencia.

\section{- $\quad$ Áreas de interés en la colaboración}

Las áreas en las que más estarían dispuestos a colaborar con otros son las áreas sustantivas de su negocio: compras y ventas con un $57 \%$ y un $29 \%$ respectivamente; el resto se encontraba atomizado en áreas como publicidad, asesorías, almacenamiento, etcétera.

- $\quad$ Áreas de oportunidad que identifican

Los encuestados identifican tres áreas en las que sus negocios necesitarían ayuda principalmente: compras, ventas y publicidad; todos opinaron que necesitan más capital para fortalecerse, es decir, financiamientos.

\section{- $\quad$ Preferencias para recibir asesorías}

El 49\% consideró que sería mejor recibir asesoría de una agrupación de comerciantes para fortalecer su negocio, el 22\% opinó que del gobierno, el 19\% prefería a una universidad y el resto no lo sabía. Los elementos que los hicieron responder de esa forma fueron: la confianza, la experiencia y la capacitación. 


\section{- Perspectiva de contar con una asociación de comerciantes}

El 51\% de los comerciantes encuestados se manifestó a favor de que existiera una agrupación que sirviera para fortalecerse económicamente, el 25\% opinó abiertamente que no funcionaría y el resto se abstuvo de opinar.

Al revisar otras variables reportadas por INEGI, tales como la situación socioeconómica de los habitantes, quienes finalmente son los dueños de las microempresas, se detectó que en 1995 el 67\% de la población de Valle de Chalco Solidaridad era menor de 30 años y en 2000 se encontraban en este grupo de edades el 69\% de la población. Las personas con 12 años de edad y más en 2000 representaban el 68\% de la población total del municipio.

La población económicamente activa (PEA) en 2000 era de 35.26\%, de la cual el 98\% se encontraba ocupada. De acuerdo con el INEGI, en ese mismo la población mayor de 12 años económicamente inactiva era de 32\% de la población total del municipio, por lo tanto, la otra tercera parte correspondía a personas menores de 12 años quienes no entran en los parámetros de actividad o inactividad económica.

Los motivos de inactividad económica del 32\% de la población eran: porque se dedicaban a los quehaceres del hogar $50 \%$, porque eran estudiantes $31 \%$, porque tenían otro tipo de actividad $17 \%$, porque eran jubilados, pensionados o incapacitados permanentemente para el trabajo $2 \%$.

La población que asistía a la escuela y que tenía entre 6 y 24 años de edad hasta el año 2000 era de 29.55\%. Según datos del INEGI el grado promedio de escolaridad de la población municipal era de 7 años ${ }^{12}$. La población que no asistía a la escuela, también entre los 6 y los 24 años de edad, en 2000 era de 17.22\%. El nivel de analfabetismo a ese mismo año de las personas que se encontraban en el rango de edades entre 6 y 14 años era de 1.8\% de la población total del municipio.

El 70\% de la PEA ocupada en 2000 era empleado u obrero, el 22\% era trabajador por su cuenta, $5 \%$ era jornalero, patrón o trabajador sin pago y el 3\% restante no especificó.

${ }^{12}$ www.inegi.gob.mx, 2000. 
Tabla 7

Distribución de la PEA ocupada por sectores (2000)

\begin{tabular}{|c|c|c|}
\hline \multirow[t]{2}{*}{ Secter } & \multicolumn{2}{|c|}{ Valle de Cbalce } \\
\hline & Persenas & $\%$ \\
\hline $\begin{array}{l}\text { Agricultura, ganaderia, apeovechamiente forestal, } \\
\text { pesca y caza }\end{array}$ & 401 & $0.3 \%$ \\
\hline Mineria & 39 & $0.03 \%$ \\
\hline Electricidad y aqua & 288 & $0.2 \%$ \\
\hline Construeción & 13,757 & $12.3 \%$ \\
\hline Indusirias manufactureras & 23.877 & $21.3 \%$ \\
\hline Comercio & 27,729 & $24.8 \%$ \\
\hline Transporte, sorreos y almacenamiento & 6.059 & $5.4 \%$ \\
\hline Informacion en medies masives & 608 & $0.5 \%$ \\
\hline Servicios financienos $y$ de segunes & 339 & $0.3 \%$ \\
\hline $\begin{array}{l}\text { Servicios inmobiliarios y de alquiler de bients } \\
\text { nuebles }\end{array}$ & 314 & $0.3 \%$ \\
\hline Servicios profexicales & 1.124 & $1.0 \%$ \\
\hline Servicios de apoyo a negocilos & 4509 & $4.0 \%$ \\
\hline Servicios educativos & 2.317 & $2.0 \%$ \\
\hline Servicios de salud y asistencia social & 1.896 & $1.7 \%$ \\
\hline Servicios de esparcimiente y cultarales & 649 & $0.6 \%$ \\
\hline Servicios de hoteles y restaurantes & 6.256 & $5.6 \%$ \\
\hline Otros servicios excepto gobieme & 12.932 & $11.5 \%$ \\
\hline Actividades del gobierno & 5.916 & $4,4 \%$ \\
\hline No especificade & 3.603 & $3.2 \%$ \\
\hline TOTAL & 111.503 & $100 \%$ \\
\hline
\end{tabular}

Fuente: Elaboración propia con datos del INEGI, 2002

El 24\% de la PEA ocupada laboraba en 2000 en el sector comercio, el 21\% en industrias manufactureras, el $12 \%$ en la construcción, el $11 \%$ en otros servicios excepto gobierno. El 32\% restante se encontraba atomizado en sectores que van desde el agrícola hasta el de servicios de hoteles y restaurantes.

Otra variable importante en la investigación es la presencia de instituciones de educación superior, por ser ellas quienes tienen en sus manos las posibilidades reales de realizar capacitación, asesoría, consultaría, transferencia de conocimientos, etc. para mejorar el desempeño de los empresarios de la región.

\section{Características de las instituciones de educación superior en el municipio}

En Valle de Chalco Solidaridad sólo existe una institución de educación superior (IES), la Universidad Autónoma del Estado de México, que a través de su Centro Universitario imparte seis licenciaturas, dos de las cuales corresponden al área económico administrativa, otra al campo de las ciencias sociales, una al campo de la salud, otra al ámbito de la ingeniería y una más que corresponde el área de la arquitectura y el diseño. 
Para contar con mayores elementos en lo que respecta a la descripción de esta variable, se optó por indagar en otras IES de la región; se encontraron dos más en el municipio de Chalco, una de capital privado y otra de patrimonio público.

El Centro de Estudios Superiores Azteca y el Centro Universitario UAEM Valle de Chalco son las dos instituciones que cuentan con licenciaturas afines al área de los negocios. La antigüedad en la impartición de estas licenciaturas es superior a cinco años en ambos casos.

\section{Tabla 8}

\section{Instituciones de Educación Superior y licenciaturas que imparten (2005)}

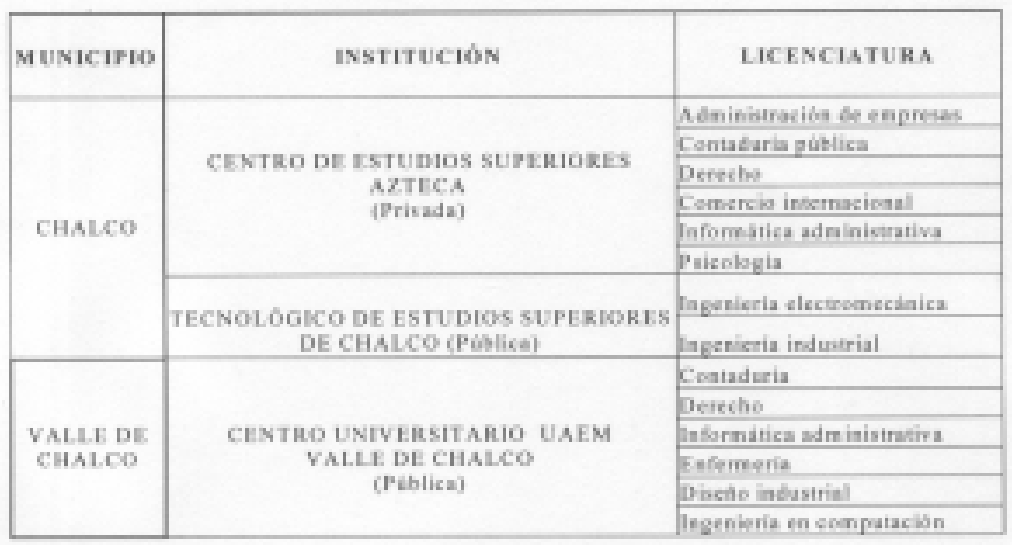

Fuente: Elaboración propia con datos proporcionados por cada Institución

De acuerdo con los resultados de la entrevista practicada a sus directores, se detectó:

\section{- $\quad$ Experiencia en investigación relativa a empresas}

Las dos instituciones señaladas anteriormente realizan investigación, aun cuando están en sus inicios; los temas de investigación en las dos instituciones son relativos a educación y desarrollo empresarial; en una de ellas se cuenta además con un proyecto de ingeniería y tecnología; una de las dos instituciones ofrecerá próximamente estudios de posgrado en Contaduría, Derecho y Psicología. Ambas instituciones consideraron que es necesario tomar en cuenta, dentro de su misión y visión institucionales, el satisfacer las necesidades de la sociedad. 
- $\quad$ Participación en el desarrollo económico de la región

Estas mismas instituciones (El Centro de Estudios Superiores Azteca y el Centro Universitario UAEM Valle de Chalco) coinciden en que es necesario interactuar con los empresarios de la zona, a través de asesorías, consultoría, desarrollo de proyectos conjuntos, etc., por lo que cuentan con programas de vinculación y convenios firmados con instituciones tanto del sector público como del privado para lograr sus metas.

- $\quad$ Experiencia en programas gubernamentales de desarrollo empresarial

Las dos instituciones cuentan con experiencia en la participación de proyectos de esta índole, citaron como ejemplos el "Programa de modernización de la empresa" o el de "Jóvenes empresarios", y ambas estarían dispuestas a participar nuevamente en éstos o algún otro programa de desarrollo empresarial, incluso asumiendo el papel de líder del equipo de trabajo. Dentro de los beneficios inmediatos que visualizan de participar en estos proyectos son un crecimiento para su institución educativa y para la economía regional, así como una mejor vinculación de sus docentes y estudiantes con problemas y oportunidades reales del entorno.

\section{- $\quad$ Disposición para colaborar entre sí}

Las dos instituciones estarían totalmente dispuestas a colaborar con otras instituciones de educación superior de la zona para fomentar el desarrollo empresarial de la región.

\section{- $\quad$ Responsabilidad ante la comunidad}

La institución privada considera que su responsabilidad es la de brindar formación universitaria y de bachillerato a personas de bajos recursos y que en el futuro su papel será profesionalizar los servicios que ofrece. La institución pública considera que su papel es brindar formación profesional en seis licenciaturas, así como marcar la presencia de la universidad pública en la sociedad con oferta cultural, educativa y de asesorías. En el futuro, la formación profesional que imparta se centrará en el área de la salud, el diseño y la programación.

\section{- $\quad$ Dirección de la vinculación con su entorno}

La institución privada ha buscado relacionarse con otras instituciones privadas de la zona oriente del Estado de México, la institución pública ha favorecido los vínculos al interior de la misma Universidad a través de sus 
distintos centros universitarios, sobre todo con aquellos de la zona oriente del Estado de México. La experiencia en participación en proyectos desarrollados con otra institución es distinta: la institución privada se ha enfocado un poco más a desarrollar proyectos con asociaciones de instituciones educativas privadas, la institución pública ha participado en proyectos más concretos tanto con instituciones educativas de nivel medio superior como con empresarios de la región y personal del H. Ayuntamiento de Valle de Chalco Solidaridad.

\section{- $\quad$ Evaluación de los resultados obtenidos en proyectos de vinculación}

La institución privada considera que no fueron tan buenos como se hubiera querido, la institución pública considera que sí fueron satisfactorios y que fueron de gran relevancia por la seguridad que se obtuvo al participar en ellos. Sin embargo, ambas coinciden en que los elementos necesarios para mantener o mejorar los resultados son: contar con una mayor asesoría técnica, certificar a los instructores, lograr una mayor comunicación con los clientes (empresas, ayuntamiento, etc.), contar con más apoyo económico y buscar una mayor interacción con las IES de la zona.

\section{- Habilitación para participar en un proyecto de asociación}

En cuanto a participar como líderes en un proyecto conjunto con IES de la zona para fomentar el desarrollo económico, la institución privada considera estar totalmente preparada, la institución pública considera que lo podría lograr pero con algunas carencias: en infraestructura y de personal.

Los programas de apoyo en los que se sustentaría el proyecto de asociación empresarial se describen en el siguiente apartado.

\section{Programas de apoyo gubernamentales que contemplan estrategias de integración al año 2006}

Desde el documento máximo de planeación del país, el Plan Nacional de Desarrollo, en el periodo 2001 -2006 se establece:

en su apartado 6 denominado 'Área de Crecimiento con Calidad', como uno de los 
objetivos rectores del propio Plan, el elevar y extender la competitividad del país, a través de las siguientes estrategias:

- $\quad$ Promover el desarrollo y la competitividad sectorial

- $\quad$ Formación de recursos humanos y una nueva cultura empresarial

- $\quad$ Promover el uso y aprovechamiento de la tecnología

- Instrumentar una política integral de desarrollo empresarial

- Otras $^{13}$

Por su parte, el Programa de Desarrollo Empresarial (PDE) 2001-2006, establece que:

las acciones del mismo, están diseñadas para cumplir con las metas siguientes:

- Atender un millón trescientas mil empresas.

- Incrementar el valor de la producción de las MIPYMES a una tasa promedio del 5 por ciento anual en términos reales.

- Disminuir eficientemente el 5 por ciento del valor de las importaciones como porcentaje total del PIB.

- Incrementar la integración de insumos nacionales en 5 puntos porcentuales para las exportaciones manufactureras no maquiladoras y en 20 por ciento para las exportaciones de la industria maquiladora.

- Incorporar a personas en condición de extrema pobreza al desarrollo nacional, a través de la generación de empleos productivos.

- Consolidar una red de multiplicadores y facilitadores de apoyo extendida en todos los municipios y entidades federativas del país, en donde los organismos empresariales, las instituciones educativas y de investigación tengan un papel primordial, apoyadas por todas las instancias de gobierno. ${ }^{14}$

Dentro de sus estrategias, el PDE incluye el fortalecimiento local y por sector económico a través de un modelo de integración que es el de las cadenas productivas, para este programa "[...] las entidades federativas y los municipios son la base del desarrollo económico del país. Estos deberán ser generadores e impulsores de la participación de los diferentes agentes económicos y sociales [...]."15

\footnotetext{
${ }^{13} \mathrm{http} / / / 204.153 .24 .126 /$ fondopyme/default2.asp, 2005.

${ }^{14}$ Idem.

${ }^{15}$ Idem.
} 
De manera particular el Fondo PYME establece las figuras de desarrollo sectorial y regional como una de sus pociones para hacer más competitivas a las MIPYMES (ver anexo 3). Adicionalmente a estos programas de carácter federal, se hizo una revisión de los programas estatales para tener una visión más completa de los apoyos existentes en materia de integración, sin embargo sólo uno de los programas establece un modelo de integración: las cooperativas (ver anexo 4).

Tabla 9

Programa de Desarrollo Empresarial: estrategias y ejes de acción

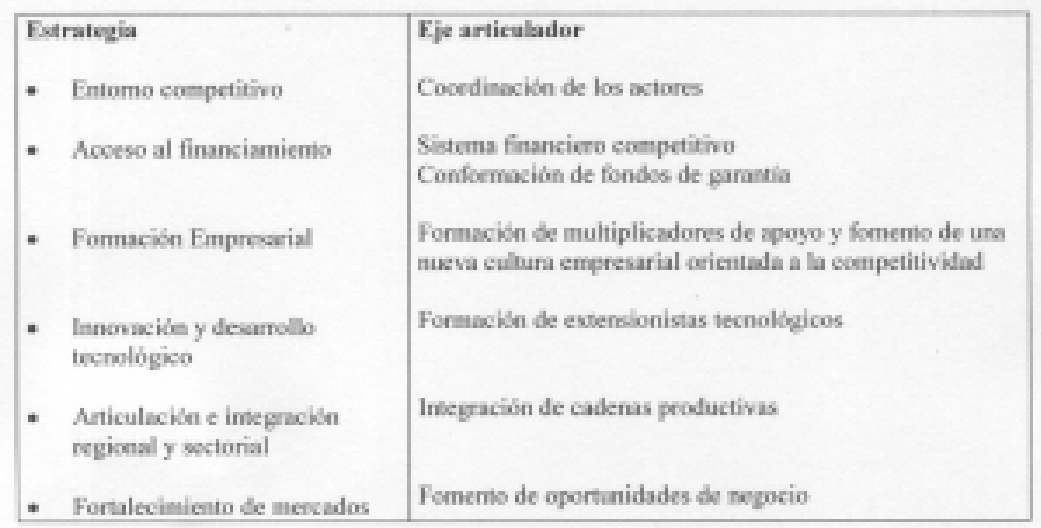

Fuente: Secretaria de Economia. 2005

Derivado del análisis de todas las variables antes descritas, fue posible realizar un análisis de los factores a favor y en contra del proyecto de asociación, en la siguiente página se presenta un resumen del análisis.

$\mathrm{Al}$ analizar los elementos en su conjunto se aprecia que hay variables que encuentran más elementos desfavorables que otras y viceversa, pero es posible determinar que sí existen los elementos suficientes para realizar proyectos de asociación en el municipio considerando la información que aportaron estas variables.

\section{Conclusiones}

Elementos a favor: Se demuestra que sí existe intención de participar en proyectos de colaboración empresarial, el 62\% de los empresarios dedicados al comercio así lo manifestaron. Las dos IES de la región estarían dispuestas a participar como 


\section{Elementos de integración de microempresas comerciales en el oriente del Estado de México en los primeros años del siglo XXI}

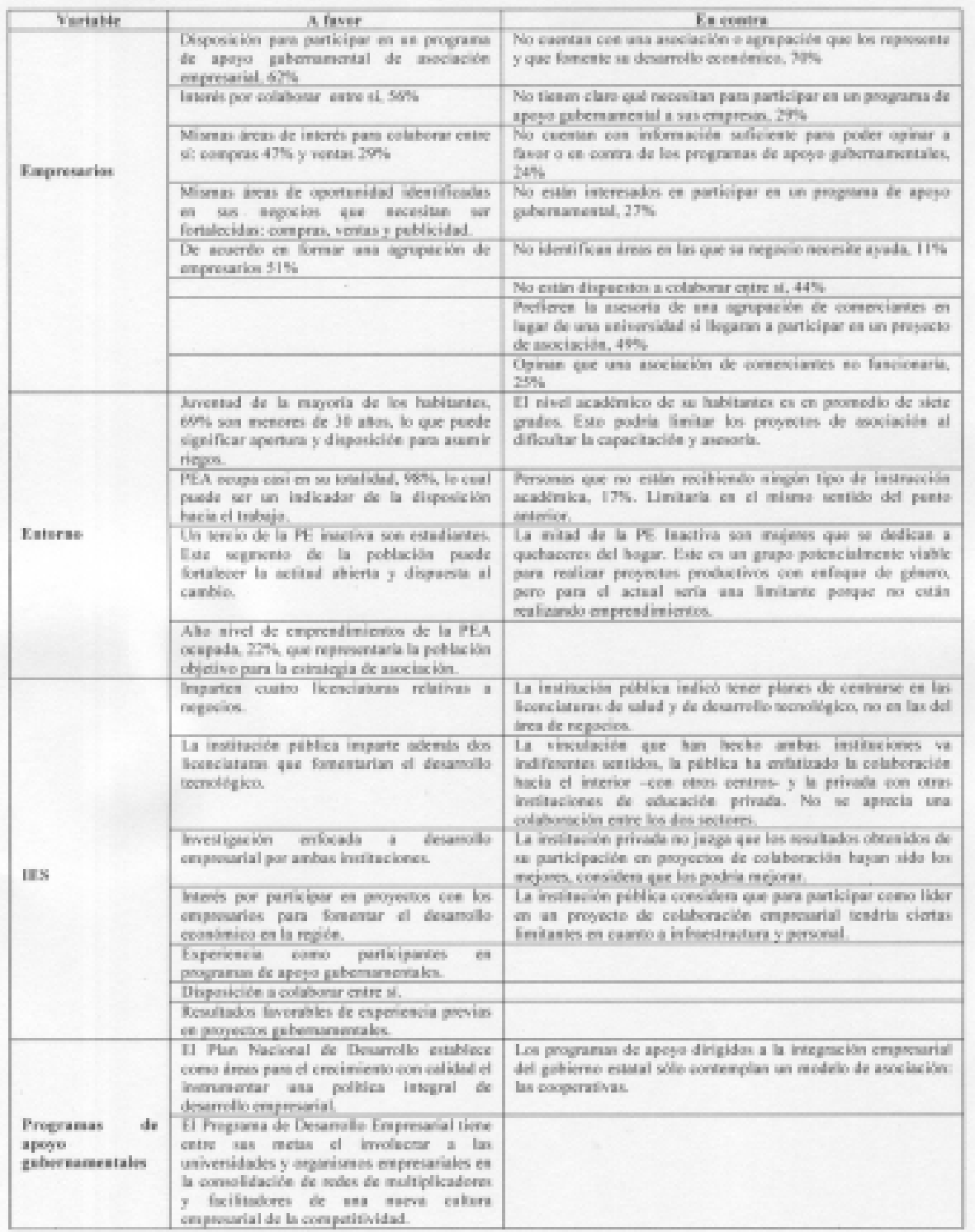


asesoras o líderes de proyectos de integración, aportando el marco institucional del modelo; una de ellas tendría que resolver previamente sus limitantes de infraestructura y personal. El Fondo PYME establece dentro de sus líneas de apoyo a las estrategias de asociación empresarial y a las IES como líderes. La función de la universidad sería en primera instancia difundir la mecánica de operación de los proyectos, requisitos y ventajas para posteriormente establecer el mecanismo de asociación en conjunción con los empresarios mejor informados con una visión más clara de su propia problemática y de las posibles alternativas de solución.

Elementos en contra: La falta de claridad en el compromiso que implicaría obtener apoyos gubernamentales para lograr su desarrollo económico que se deriva de la falta de claridad de su propia problemática y sus alternativas de solución. Sólo un 19\% de los encuestados aceptarían la integración de una IES en el modelo, preferirían una organización empresarial como organismo intermedio, lo cual representaría un trabajo doble para la universidad, ya sea organizar y capacitar previamente para lograr su agrupación y posteriormente proponer el modelo de asociación. Si se trabajara un proyecto de asociación con fondos estatales la única figura de asociación que se podría adoptar sería la cooperativa. La intención de la institución pública de estudios superiores es centrarse en áreas del conocimiento en primera instancia distintas al desarrollo empresarial.

\section{Recomendaciones}

1. Se debe orientar el apoyo necesario para este segmento de comercios que tienen la posibilidad de aumentar su escala y, por tanto, el empleo formal y permanente en la zona de Valle de Chalco. El programa que los fortalecerá e integrará debe contemplar la generación de una red de cooperación que los beneficie en la reducción de costos para que aumente los beneficios sin olvidar que la reducción de costos tiene un límite inherente a la naturaleza de la actividad comercial.

2. El modelo debe considerar el intercambio de información en áreas prioritarias: almacén, distribución de planta, canales de distribución, ventas, compras y planeación estratégica. La creación de las políticas y reglamentaciones al interior de la organización que permita un mejor control de ésta; este programa debe implantarse con la cooperación de las empresas involucradas y el aprovechamiento de los programas existentes creados por el gobierno. 
La universidad tiene un papel fundamental: generar los programas de apoyo empresarial integral para que la asociación se consolide y pueda beneficiar a las empresas familiares.

3. Debe buscarse una alternativa que propicie un efecto mayor sobre la creación de empleos, integración y consolidación de cadenas productivas u otra figura de integración de las principales empresas en la zona, vinculadas a la cooperación del gobierno local y a la universidad.

4. La gran cantidad de mujeres inactivas económicamente por dedicarse a labores del hogar podría ser un área de oportunidad propicia para el desarrollo de proyectos comunitarios de mujeres como algunos que se han desarrollado con éxito a través del Banco Compartamos, caso del Grupo Solidario La Esperanza en el estado de Tabasco. ${ }^{16}$

5. Se deberán integrar pequeños núcleos de comerciantes que recibirán capacitación por parte de la universidad, quienes diseñarían al término de su capacitación estrategias de mejora de su negocio en lo individual y como grupo.

6. Se buscaría que estos mismos comerciantes, con apoyo de la universidad, fueran los replicadores de la capacitación con pequeños núcleos de comerciantes del municipio en un esquema similar al que operan las compañías "multinivel"; es decir, un comerciante atrae a tres o más consiguiendo beneficios extras mientras más comerciantes atraiga a la capacitación; los beneficios podrían traducirse en horas de capacitación sin costo en áreas que el comerciante determine necesarias.

7. Una vez ampliado el círculo de microempresarios, se podría plantear la propuesta de organizar una asociación de comerciantes, que en primera instancia serían los integrantes de este primer equipo y posteriormente se irían incorporando más integrantes.

8. El objetivo inicial de la asociación sería en primera instancia el proporcionar asesoría, una vez que la asociación se fortaleciera se podría ampliar la gama de servicios hacia las necesidades que los propios asociados observaran en sus negocios.

${ }^{16}$ Francisco Payró, "De la calle al mercado", Letras libres, 2006. http://www.letraslibres.com/index.php?sec=3\&art=11158\%22 
Elementos de integración de microempresas comerciales en el oriente del

Estado de México en los primeros años del siglo XXI

\section{Bibliografía}

CARRILLO, Jorge y Alfredo Hualde, "Desarrollo Regional y Maquiladora Fronteriza: Peculiaridades de un Cluster Electrónico en Tijuana", en la revista El mercado de valores, núm. 10/octubre, México, Nacional Financiera, 2000.

HERNÁNDEZ SAMPIERI, Roberto (2003), Metodología de la investigación, $3^{\text {a }}$ ed., México, Mc Graw Hill.

Ley para el desarrollo de la competitividad de la micro, pequeña y mediana empresa, México, 2005.

LOYOLA ROCHA, Jaime (2002), Historias para ser contadas a los recién llegados, Municipio de Valle de Chalco Solidaridad, México.

MEYER-STAMER, Jörg (2000), "Estrategias de Desarrollo Local y Regional: Clusters, Política de Localización y Competitividad Sistémica", en la revista El mercado de valores, núm. 9/septiembre, México, Nacional Financiera.

RUEDA PEIRO, Isabel et al. (1997), Las empresas integradoras en México, Siglo XXI, México.

SCHMITZ, Hubert (2000), "¿Tiene importancia la cooperación Local? Experiencias de clusters industriales en el sur de Asia y América Latina", en El Mercado de Valores, No. 9/septiembre, Nacional Financiera, México.

\section{Páginas electrónicas}

www.contactopyme.gob.mx, 2005.

www.edomex.gob.mx, 2004

www.inegi.gob.mx., 2002.

www.letraslibres.com, 2006.

www.monografías.com, 2006.

www.prd.usp.br, 2002.

www.se.gob.mx, 2002. (A) 


\section{Anexos}

Anexo 1. Cuestionario para las microempresas comerciales de Valle de Chalco

Este cuestionario tiene como OBJETIVO: Detectar el interés de las empresas comerciales de Valle de Chalco en participar en un modelo de asociación empresarial para fortalecerse económicamente.

Instrucciones: Conteste objetivamente las preguntas que a continuación se le formulan.

\section{A. INFORMACIÓN GENERAL}

\# de negocio:

1. Nombre del negocio:

2. Número promedio de empleados al año:
( ) 0 - 2
( ) 3 - 5
( ) 6 - 8
( ) 9 o más

3. ¿Su negocio está afiliado a alguna agrupación de comerciantes de la zona?
( ) Sí
( ) No (Pase a la pregunta 6)

4. Nombre de la agrupación.

5. Marque los servicios que le brinda su agrupación:
( ) Asesoría fiscal
( ) Préstamos de dinero
( ) Gestión de trámites

( ) Cursos en general ( ) Otros

6. ¿Participa actualmente en algún programa de apoyo para fortalecer a los pequeños negocios?

( ) Sí

( ) No (Pase a la pregunta 8)

7. ¿Cuál? (Pase a la pregunta 10)

8. ¿Qué necesita para participar en un programa de apoyo a los pequeños negocios?

9. Contando con información suficiente, estaría usted interesado en participar en algún programa de apoyo del gobierno federal, estatal o municipal para fortalecer a su negocio?
( ) Sí ¿Por qué?
( ) No ¿Por qué?

\section{B. OPERACIONES}

10. El volumen mensual de sus compras de mercancía son de:

( ) Menos de $\$ 1,000$ ( ) Más de \$1,000 pero menos de \$3,000 ( ) Más de \$3,000 pero menos de \$5,000 ( ) Más de \$5,000 pero menos de \$8,000 ( ) Más de \$8,000

11. Los gastos mensuales de traslado de esa mercancía son de:

（ ) Menos de $\$ 300$ （） Más de $\$ 300$ pero menos de $\$ 500$ （） Más de $\$ 500$ pero menos de $\$ 800$

( ) Más de $\$ 800$ pero menos de $\$ 1,200$ ( ) Más de $\$ 1,200$

12. ¿Cuál es el mecanismo de contabilidad de utiliza para registrar sus operaciones?

( ) No llevo contabilidad （ ）A través de un cuaderno ( ) Con la ayuda de un contador 
Elementos de integración de microempresas comerciales en el oriente del

Estado de México en los primeros años del siglo XXI

\section{ASOCIACIÓN}

13. Si tuviera que elegir, ¿qué tan dispuesto estaría en colaborar con otros negocios similares al suyo para fortalecerse económicamente?

( ) Dispuesto ¿¿Por qué?

( ) No dispuesto ¿Por qué?

14. ¿En que actividades estaría dispuesto a colaborar con otros negocios? Marque todos los paréntesis que le interesen.
( ) Compras
( ) Publicidad
( ) Almacenamiento
( ) Compartir equipo
( ) Ventas
( ) Capacitación
( ) Financiamiento
( ) Asesorías

15. ¿En que aspectos considera que su negocio necesita apoyo para obtener un mejor crecimiento económico? Marque todos los paréntesis que considere necesarios.
( ) Compras
( ) Ventas
( ) Almacenamiento
( ) Financiamiento
( ) Compartir equipo ( ) Asesorías
( ) Capacitación
( ) Publicidad

16. ¿En un programa de apoyo para fortalecer a las micro, pequeñas y medianas empresas, quien preferiría que lo asesorara?
( ) Universidad ( ) Agrupación de comerciantes
( ) Gobierno Federal, Estatal o Municipal
( ) Otro ¿Cuál?
17 ¿Por qué?

18. De los siguientes impuestos señale los pagos que realiza:
( ) Impuestos municipales ( ) Impuestos federales
( ) Licencias y permisos

19. ¿Qué opina usted de que los comerciantes de Valle de Chalco contaran con una asociación que les brindara apoyo para fortalecerse económicamente?

Gracias por su apoyo. 


\section{Anexo 2. Guía de entrevistas a las IES de la zona de estudio.}

La presente entrevista tiene la intención de conocer el perfil y trayectoria de las Instituciones de Educación Superior (IES) de Chalco y Valle de Chalco, en cuanto a su vinculación con el entorno.

Nombre de la Institución:

Domicilio:

Nombre del director o coordinador:

Fecha:

\section{Información General}

1. ¿Podría usted decir qué licenciaturas se imparten en la Institución de Educación Superior (IES) que usted dirige y por cuántos años se han impartido?

2. ¿Cuál es la zona de influencia de esta Institución?

3. ¿Se imparten estudios de posgrado en esta Institución? ¿Cuáles?

4. ¿La IES que usted dirige realiza investigación? ¿en qué áreas del conocimiento y cuánto tiempo llevan realizándola?

5. Si realiza investigación, indique de qué índole es: educativa, de ciencias básicas, de vinculación con el entorno, etc. Visión

6. ¿Cuál es la visión de esta IES en el mediano plazo en cuanto a la vinculación con su entorno?

7. ¿En el mediano plazo cómo visualiza a esta IES en su relación con los diferentes niveles de gobierno (municipal, estatal, federal)?

8. ¿En un plazo no mayor de cinco años en que programas de fortalecimiento empresarial visualiza participando a esta IES?

9. ¿Actualmente cuál es el papel que desempeña esta IES en su entorno? ¿Será el mismo que desempeñará en cinco años? Explique.

\section{Vinculación}

10. ¿Qué proyectos desarrolla o ha desarrollado esta IES con otras IES de la región?

11. ¿Qué proyectos puede mencionar en los que participe o haya participado esta IES con alguna otra entidad, sea educativa o no?

12. ¿Existe un plan formal de vinculación de esta IES? ¿Cuál es su principal objetivo?

13. ¿Ha participado esta IES en algún programa de apoyo empresarial ya sea del gobierno federal, estatal o municipal? Indique en cuál.

14. ¿Cómo calificaría usted los resultados obtenidos de la participación de esta IES?

15. ¿Qué necesitaría para que los resultados obtenidos fueran mejores?

16. ¿Cómo calificaría los programas o proyectos? ¿Estaría dispuesto a participar nuevamente?

17. ¿Cómo calificaría la infraestructura, personal, conocimientos, etc. de esta IES para participar como líder de un proyecto de desarrollo empresarial auspiciado por el gobierno federal?

18. Independientemente de su respuesta de la pregunta anterior, ¿estaría interesado en que esta Institución asumiera ese liderazgo?

19. ¿Qué beneficios visualiza si se diera esa participación?

20. ¿Estaría interesado en unir esfuerzos con otras IES de la región para fomentar el desarrollo empresarial de la zona? Explique cómo.

Gracias por su atención. 
Elementos de integración de microempresas comerciales en el oriente del

Estado de México en los primeros años del siglo XXI

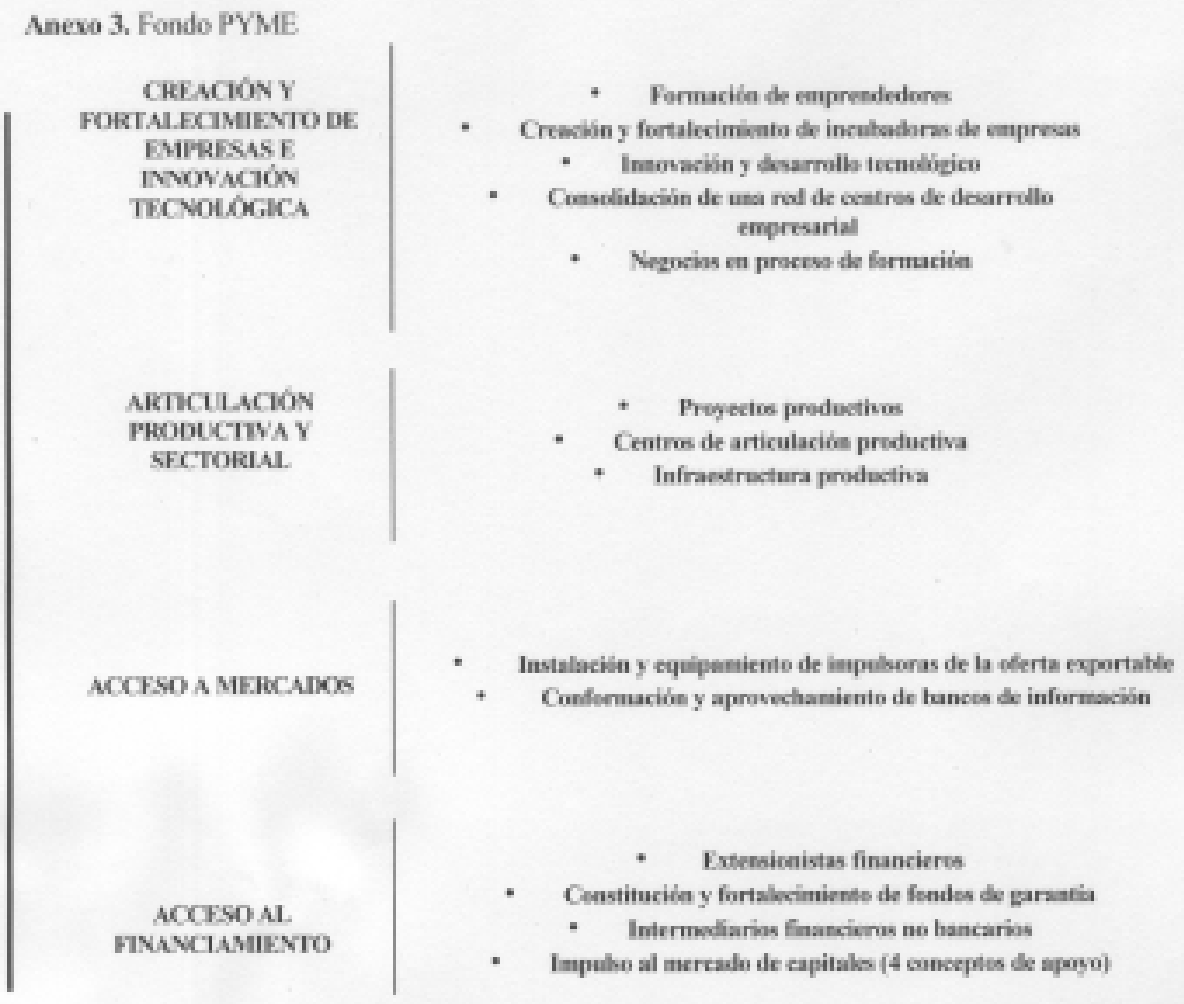

Fuente: Secretaria de Economia, 2005 
Nidia López Lira

\section{Anexo 4. Programas estatales}

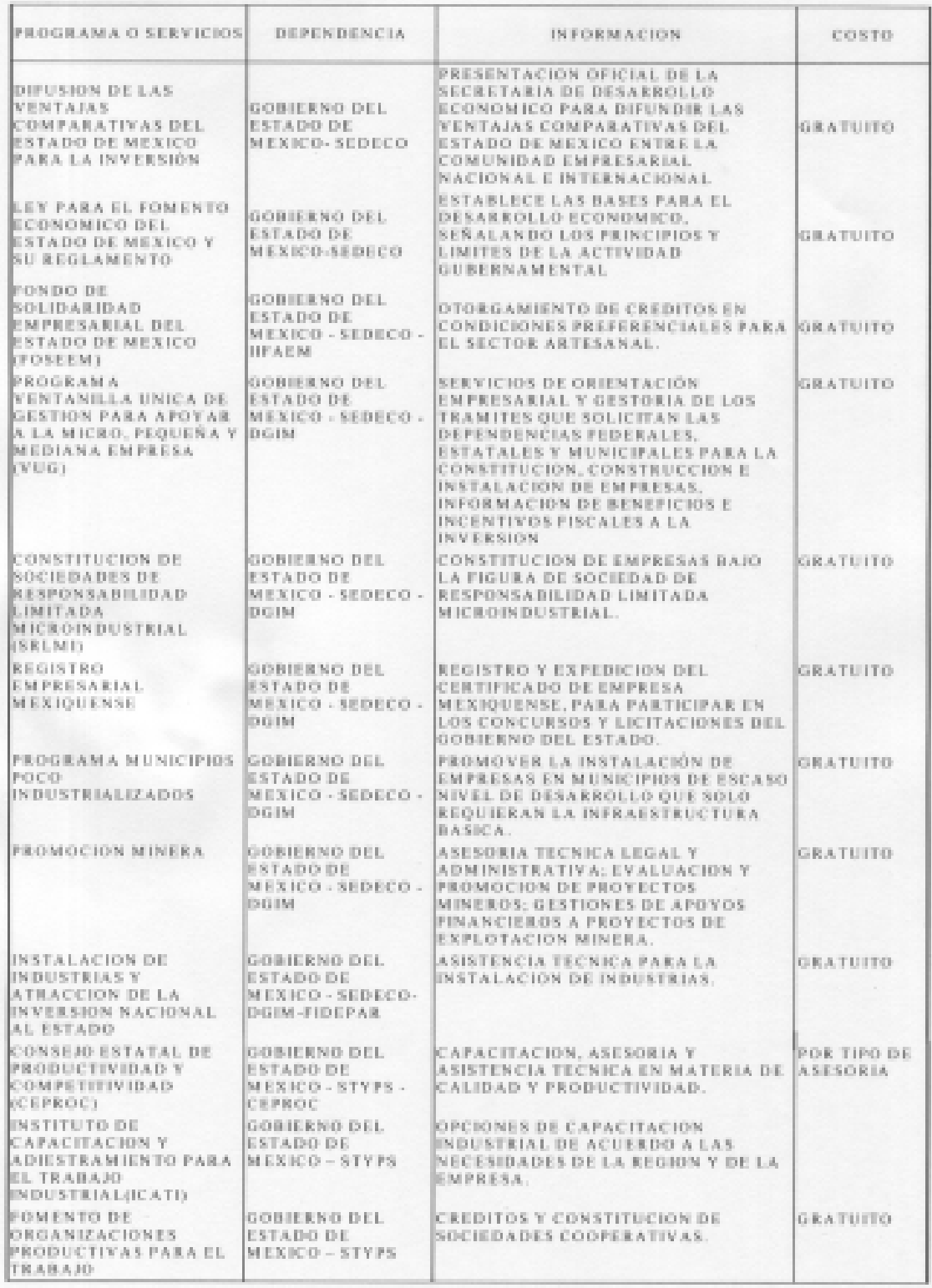

Fucnic: Gobiereso del Exrado de Mrixico, 2004 